\title{
Epidemiologia e impacto econômico da ocorrência da brucelose em um município do
}

\section{Estado de Santa Catarina}

\author{
Epidemiology and economic impact of brucellosis in the municipality of Santa Catarina State, \\ Brazil
}

Epidemiología e impacto económico de la ocurrencia de la brucelosis en una ciudad de la unidad federativa de Santa Catarina, en Brasil

\author{
Marina Gabriela Possa \\ ORCID: https://orcid.org/0000-0002-9463-4666 \\ Universidade Federal da Fronteira Sul, Brasil \\ E-mail: marinagaabriela@ hotmail.com \\ Fabricio Bernardi \\ ORCID: https://orcid.org/0000-0002-4449-962X \\ Universidade Federal da Fronteira Sul, Brasil \\ E-mail: bernardi_fabricio@hotmail.com \\ Adalgiza Pinto Neto \\ ORCID: https://orcid.org/0000-0002-2180-2390 \\ Universidade Federal da Fronteira Sul, Brasil \\ E-mail: adalgiza.neto@uffs.edu.br \\ Jonatas Cattelam \\ ORCID: https://orcid.org/0000-0002-4395-2189 \\ Universidade Federal da Fronteira Sul, Brasil \\ E-mail: jonatas.cattelam@uffs.edu.br \\ Iucif Abrão Nascif Júnior \\ ORCID: https://orcid.org/0000-0001-8612-612X \\ Universidade Federal da Fronteira Sul, Brasil \\ E-mail: iucif.junior@uffs.edu.br \\ Camila Keterine Gorzelanski Trenkel \\ ORCID: https://orcid.org/0000-0001-7614-1473 \\ Universidade Federal da Fronteira Sul, Brasil \\ E-mail: catrenkel@gmail.com \\ Marcelo Falci Mota \\ ORCID: https://orcid.org/0000-0002-4739-0910 \\ Universidade Federal da Fronteira Sul, Brasil \\ E-mail: marcelo.mota@uffs.edu.br
}

\begin{abstract}
Resumo
A brucelose é uma das mais importantes e negligenciadas doenças zoonóticas. Objetivou-se com o estudo conhecer a epidemiologia, as perdas econômicas e os fatores de risco da brucelose em bovinos, equinos, cães e humanos no município de Flor do Sertão - Santa Catarina. Examinou-se para Brucella abortus, entre 2017 e 2018, 2.750 bovinos, de 131 propriedades; 20 equinos e 29 cães. Os testes sorológicos de triagem seguidos de confirmatório realizados foram o AAT e 2 ME/SAT. Para casos de brucelose humana, a secretaria de saúde do município concedeu 206 resultados sorológicos referentes ao mesmo período de estudo dos bovinos. Foram calculadas prevalências da doença para as populações e utilizado o teste da árvore de decisão, pelo programa estatístico Weka ${ }$, para estabelecer associação entre fatores de risco e a positividade de rebanhos. O teste de Spearman (GraphPad Prism®) foi utilizado para estabelecer correlação entre casos humanos e bovinos. 45,5\% da população de bovinos do município foi testada, demonstrando prevalência de brucelose de 0,29\%. As perdas econômicas anuais foram de R \$ 14.747,92. Foram positivos $5,33 \%$ dos humanos testados. Os fatores associados com a positividade para a doença baseiam-se no consumo produtos lácteos sem tratamento térmico, e prevalência de brucelose acima de zero. Observou-se que em Flor do Sertão - SC, a prevalência de brucelose em bovinos é baixa, porém, elevada para focos. No entanto, a prevalência da doença em humanos foi elevada, sendo superior a prevalência animal, possivelmente decorrente dos fatores de risco associados a positividade da doença, como o consumo de lácteos crus.
\end{abstract}

Palavras-chave: Brucelose; Perdas produtivas; Perdas reprodutivas; Saúde pública; Zoonose. 


\begin{abstract}
Brucellosis is one of the most important and neglected zoonotic diseases. Thus, the objective of this work was to describe the epidemiology, economic losses, and risk factors for brucellosis in bovine cattle, horses, dogs, and humans in the municipality of Flor do Sertão, state of Santa Catarina, Brazil. Exams for Brucella abortus were conducted from 2017 to 2018 in 2,750 bovines from 131 farms, 20 horses, and 29 dogs. Serological screening tests were used, followed by confirmatory tests (AAT and 2ME/SAT). Regarding the cases of brucellosis in humans, the municipality's health department provided 206 serological results that were recorded in the same period of the study of cattle brucellosis. The prevalence of the disease for the populations was calculated and the decision tree test was applied using the $\mathrm{Weka}^{\circledR}$ statistical program to establish an association between risk factors and the positivity in the herds. The Spearman test (GraphPad Prism-) was used to establish a correlation between brucellosis cases in humans and in bovine cattle. The tests encompassed $45.5 \%$ of the cattle population in the municipality, showing a prevalence of brucellosis of $0.29 \%$. The annual economic losses were R\$ (BRL) 14,747.92. The percentage of humans that tested positive for brucellosis was 5.33\%. The factors associated with positivity for the disease were based on the consumption of dairy products without heat treatment, and prevalence of brucellosis above zero. The prevalence of brucellosis in bovine cattle in Flor do Sertão is low, but high for local outbreaks. However, the prevalence of the disease in humans was high, which were higher than the prevalence in animals, probably due to the risk factors associated with the positivity of the disease, such as the consumption of raw dairy products.
\end{abstract}

Keywords: Brucellosis; Productive losses; Reproductive losses; Public health; Zoonosis.

\title{
Resumen
}

La brucelosis es una de las enfermedades zoonóticas más importantes pero la más desatendida. El objetivo del estudio fue saber la epidemiología, las pérdidas económicas y los factores de riesgo de la brucelosis en bovinos, equinos, caninos y humanos en la ciudad de Flor do Sertão, en Santa Catarina, Brasil. Entre 2017 y 2018, se analizaron 2.750 bovinos (de 131 granjas), 2.7 equinos y 29 perros para detectar la contaminación por Brucella abortus. Los análisis de muestreo sérico fueron los del antígeno acidificado tamponado y el 2-mercaptoetanol y la aglutinación sérica lenta en tubos, seguidos de pruebas confirmatorias. Para los casos de brucelosis humana, el departamento de salud de la ciudad concedió 206 resultados séricos para el mismo período de estudio de los bovinos. Se calculó la prevalencia de la enfermedad en las poblaciones, y se utilizó la prueba del árbol de decisión, mediante el programa estadístico Weka®, para establecer una asociación entre los factores de riesgo y la confirmación de la enfermedad en los rebaños. Se utilizó la prueba de Spearman (GraphPad Prism $\left.{ }^{\circledR}\right)$ para establecer una correlación entre los casos humanos y bovinos. Se analizó el $45,5 \%$ de la población bovina de la ciudad, y se indicó una prevalencia de brucelosis del 0,29\%. Las pérdidas económicas anuales fueron de BRL 14.747,92. Para el 5.33\% de los humanos analizados, el resultado fue positivo. Los factores asociados a la confirmación de la enfermedad se basan en el consumo de productos lácteos sin tratamiento térmico, y la prevalencia de brucelosis por encima de cero. Se observó que en la ciudad de Flor do Sertão, en Santa Catarina, Brasil, la prevalencia de brucelosis en bovinos es baja, sin embargo, alta para los brotes. Sin embargo, la prevalencia de la enfermedad en humanos fue alta, siendo superior a la prevalencia en animales, posiblemente debido a los factores de riesgo asociados a la confirmación de la enfermedad, como el consumo de productos lácteos crudos.

Palabras clave: Brucelosis; Pérdidas productivas; Pérdidas reproductivas; Salud pública; Zoonosis.

\section{Introdução}

A atividade agropecuária é uma das principais atividades econômicas do país (Instituto Brasileiro de Geografia e Estatística, 2017). Para o estado de Santa Catarina, as exportações de carnes de diferentes espécies representam as principais receitas, e o mercado diferenciado que o estado detém, se deve às práticas sanitárias implementadas. Para promover o desenvolvimento do setor agropecuário é necessário melhorar a sanidade animal, inclusive aquelas doenças com risco de transmissão para os humanos (Alfieri \& Alfieri, 2017). De acordo com a Organização Mundial da Saúde (1958) a brucelose pode ser classificada como antropozoonose, podendo ser transmitida de forma indireta ou direta, tornando o humano infectado acidentalmente.

A brucelose é uma das mais importantes e negligenciadas doenças zoonóticas (Corbel, 2006; Dadar; Shahali; Whatmore, 2019), causada por bactérias intracelulares facultativas do gênero Brucella. Haja vista que, em decorrência do grande número de animais que compõem o rebanho bovino brasileiro, seu maior impacto na saúde animal e saúde pública, é a brucelose bovina, a qual é causada pela espécie $B$. abortus, contudo, a brucelose trata-se de uma enfermidade infectocontagiosa de caráter crônico, que acomete diversas espécies domésticas e silvestres, inclusive o homem (Aires et al., 2018). 
Ademais, os animais infectados eliminam o patógeno principalmente através de fluidos e anexos fetais no momento do parto ou abortamento, como também, outras vias de eliminação são o leite e o sêmen, uma vez que, a mais relevante porta de entrada da infecção é o sistema digestório, cujo, a infecção inicia-se com a ingestão de alimentos ou água contaminados, como também por meio do contato com secreções contaminadas pelas bactérias do gênero Brucella (Bartoli et al., 2014).

Cárdenas, et al., (2019) relataram que dois terços dos países em desenvolvimento são enzoóticos para a doença. Os impactos causados pela brucelose na saúde pública, economia e comércio tornam a atuação diante da ocorrência em bovinos como prioritária frente a outras doenças (Cárdenas, et al., 2019). As perdas econômicas causadas, associadas à prevenção de infecção humana, justificam a adoção dos programas de controle (Clementino \& Azevedo, 2016).

No Brasil, em 2001, o Ministério da Agricultura Pecuária e Abastecimento (MAPA) instituiu o Programa Nacional de Controle e Erradicação da Brucelose e Tuberculose Animal (PNCEBT), com o objetivo de reduzir a prevalência da doença para entrar em fase de erradicação posteriormente (Brasil, 2006), atuando tanto em saúde pública como aumentando a competitividade do setor agropecuário no mercado externo. Apesar da implementação das estratégias previstas no PNCEBT, a evolução para a erradicação da doença ainda não ocorreu de modo satisfatório no país. O tamanho do rebanho bovino e bubalino do país é um desafio para o sucesso o programa (Angel, et al., 2012). O estado brasileiro que apresenta a menor prevalência de focos registrada no país é Santa Catarina, com 0,32\% em 2001 e 0,91\% em 2012 (Sikusawa, et al., 2009; Baumgarten, et al., 2016).

O município de Flor do Sertão - Santa Catarina possui 6038 bovinos, sendo 2700 vacas ordenhadas (Instituto Brasileiro de Geografia e Estatística, 2016), caracterizando as atividades agrícolas como a base econômica do município. Em 2017, foram detectados no sistema público de saúde casos de brucelose humana, despertando interesse do poder público em investigar a respeito da ocorrência da doença no município. Deste modo, foi desenvolvido um programa municipal com o mesmo objetivo do PNCEBT, com o diferencial do governo municipal subsidiar os custos da realização dos exames de brucelose nos animais do seu rebanho (Flor do Sertão, 2017). O objetivo, além da erradicação da doença no rebanho bovino, foi eliminar as fontes de infecção para humanos, e minimizar os prejuízos na produção de leite e de carne, além de contribuir para a redução dos gastos públicos com tratamento de pessoas doentes.

O objetivo deste estudo foi conhecer a epidemiologia da brucelose, as perdas econômicas decorrentes da doença e os fatores de risco da brucelose humana e animal (bovinos, equinos e cães) no município de Flor do Sertão - Santa Catarina.

\section{Metodologia}

Após aprovação no Comitê de Ética em Pesquisa com Seres Humanos (CEP) (3.008.740) e a Comissão de Ética no Uso de Animais (CEUA) (23205.003443/2018-99), o estudo transversal foi realizado no município de Flor do Sertão, localizado na Região Oeste de Santa Catarina.

Foram examinados para brucelose (Brucella abortus), entre setembro de 2017 a novembro de 2018, 2750 bovinos (2676 fêmeas e 74 machos) com idade igual ou superior a oito meses que correspondem a 45,5\% do rebanho total do Município. Os animais foram oriundos de 131 propriedades. Para obtenção do diagnóstico sorológico, foram realizados testes de triagem indiretos pelo Antígeno Acidificado Tamponado (AAT) (Microsules ${ }^{\circledR}$ ) (Brasil, 2006) conforme técnica padrão descrita por Alton et al., (1988). Animais reagentes ao teste de triagem foram submetidos ao teste confirmatório 2Mercaptoetanol (2-ME) associado à prova de soroaglutinação em tubos (SAT) em laboratório credenciado ao Ministério da Agricultura Pecuária e Abastecimento (MAPA). 
Foram realizados cálculos de prevalência para a brucelose, conforme Pereira (2008). Foi calculada a prevalência total para animais do município; prevalência nas fêmeas; prevalência nos machos; e prevalência nos focos/propriedades. Para os casos positivos, os animais foram caracterizados quanto ao sexo, idade e finalidade de produção.

Dos rebanhos testados, foram coletadas, por meio de questionários semiestruturados, informações zootécnicas referentes à propriedade, rebanho e sobre as pessoas residentes naquela propriedade. Com base nestes dados, nas prevalências encontradas para cada propriedade foco e na metodologia descrita por estudo de Lucas (2006), foram realizados cálculos para estimar o impacto econômico provocados pela brucelose, relativo às perdas produtivas e reprodutivas.

Os valores de referência para rebanhos positivos utilizados para cálculo das perdas econômicas foram: taxa de fertilidade $80 \%$; taxa de descarte por esterilidade 20\%; taxa de abortamento 25\%; taxa mortalidade perinatal $30 \%$; taxa de reposição normal dos rebanhos 20\%; taxa de reposição em rebanhos com brucelose $30 \%$ e aumento médio no intervalo de partos de 11,5 para 20 meses (Lucas, 2006). Outros valores utilizados para a base de cálculos foram: preço médio litro/leite: 1,25 (US\$ 0,30) (Cepea, 2019) (média para 2017 e 2018 para a Mesorregião Oeste Catarinense); valor vaca em lactação: R\$ 3.000,00 (US\$ 721,48); valor novilha reposição: R \$ 4.500,00 (US\$ 1.082,26); valor vaca de descarte: R\$2.000,00 (US\$ 480,99) e valor da cria: R \$ 600,00 (US\$ 144,30) (valores praticados na Região Oeste Catarinense) (Epagri, 2019).

Entre novembro de 2018 a fevereiro de 2019 foram testados para brucelose 29 cães e 20 equinos. O cálculo da amostragem baseou-se na população desses animais registrada no município. Para os equinos, há registros da existência de 20 animais no município (Instituto Brasileiro de Geografia e Estatística, 2013), os quais foram examinados em sua totalidade. A população de cães no município foi estimada com base na Pesquisa Nacional de Saúde (PNS), divulgada pelo Instituto Brasileiro de Geografia e Estatística (2013). A prevalência esperada para a ocorrência de Brucella abortus em cães foi de $2 \%$ com 95\% de intervalo de confiança. O valor de prevalência esperada foi baseado em valores de prevalência de brucelose bovina, uma vez que inexistem informações para o local do estudo em cães. A amostragem obtida através de cálculo no Statcalc (EpiInfo 3.0 ${ }^{\circledR}$ ) foi de 29 cães. As amostras foram colhidas em todos os cães das propriedades foco e nas propriedades perifoco dos bovinos, até completar a amostragem. Foi realizado o teste de triagem com AAT, como descrito para bovinos, que detecta cepas de Brucella lisas. Amostra de cães e equinos reagentes no teste de triagem foram encaminhadas para teste confirmatório do 2-ME.

Foram concedidos, com a anuência da Secretaria de Saúde do Município de Flor do Sertão, resultados sorológicos, bem como informações epidemiológicas de todos os pacientes humanos testados para brucelose nos anos de 2017 e 2018 . A colheita das amostras de sangue foi efetuada na rede de saúde municipal e os testes realizados pelo Laboratório Central de Saúde Pública - Santa Catarina (LACEN/SC). No período, foram colhidas 206 amostras (86 homens e 120 mulheres). Foi calculada a prevalência total da população humana; prevalência de homens; e prevalência de mulheres. Para a caracterização do perfil epidemiológico das pessoas acometidas pela doença considerou-se informações referentes a idade, sexo, localidade de residência, tipo de contato com material de risco e submissão a tratamento para a brucelose.

Os fatores de risco para a ocorrência da brucelose relativos à propriedade, rebanho e pessoas da propriedade foram tabulados e analisados pela técnica de Mineração de Dados, aplicando-se uma tarefa de classificação, utilizando o algoritmo J48 do programa computacional Weka ${ }^{\circledR}$, versão 3.8 para a construção de árvore de decisão. O algoritmo gera um modelo de regras utilizando a mínima informação necessária para a classificação, construindo gráfico na forma de árvore invertida, com o nó raiz, o primeiro atributo com maior poder de classificação, dividindo e classificando o objeto em regras semânticas na forma corpo (variáveis ou atributos classificatórios) e cabeça (classe como consequente). A árvore segue dividindo-se em ramos que representam condicionais semânticos lógicos E e OU para a classificação até as folhas (cabeças de regra) que representam a classificação. Cada ramo da árvore representa uma regra de classificação na forma SE (variável $x$ ) E (conectores 
entre as variáveis $y, z$. se houver) ENTÃO (consequente ou classe). Foi calculada a precisão do modelo e a acurácia verificada pela sensibilidade e especificidade a partir da matriz de contingência.

Foi utilizado o teste estatístico de Coeficiente de Correlação de Spearman, por meio do programa GraphPad Prism ${ }^{\circledR}$. O teste foi utilizado para verificar a correlação de casos humanos e bovinos de brucelose nas localidades do município do estudo. Para os grupos de populações em que houveram positivos para brucelose, foi aplicado o teste de Kruskal Wallis, por meio do programa estatístico $\mathrm{SAS}^{\circledR}$, com a finalidade de verificar possíveis variações estatísticas de prevalências entre os grupos.

\section{Resultados}

Foram examinados 2.750 bovinos com idade igual ou superior a oito meses, sendo 2.676 fêmeas e 74 machos, dentre a população total de bovinos no município de estudo (6.038) (IBGE, 2016). A amostra corresponde a 45,5\% dos animais existentes no município. Destes, foram positivos no teste de triagem e no teste confirmatório oito animais (sete fêmeas e um macho). A prevalência total encontrada no município foi de $0,29 \%(8 / 2.750)$, sendo de $0,26 \%(7 / 2.676)$ a prevalência nas fêmeas e de $1,35 \%$ (1/74) a prevalência nos machos.

$35,7 \%$ (131/367) das propriedades do município tiveram seus animais examinados. Os animais positivos foram oriundos de seis propriedades rurais diferentes. Desta maneira, a prevalência de focos encontrada no estudo foi 4,58\% (6/131). A prevalência média de animais positivos para cada propriedade foco foi 4,83\% (variação de 0,84\% a 8\%).

O perfil epidemiológico dos bovinos positivos demonstrou que a doença foi predominante nas fêmeas $87,5 \%$ (7/8) em relação aos machos. A faixa de idade dos animais acometidos variou de 21 a 79 meses. Quanto a finalidade de produção, 87,5\% (7/8) dos animais acometidos foram destinados a produção de leite e apenas 12,5\% (1/8) para a produção de carne.

O município onde o estudo foi desenvolvido é caracterizado por pequenas propriedades de agricultura familiar, que possuía, em média 35,5 animais/propriedade, produção média de 139,7 litros/leite/dia e média de 11,8 vacas lactantes por propriedade.

As perdas econômicas estimadas com base no método de Lucas (2006), demonstraram que a redução na produção de leite para cada propriedade foi de 2,3\%; 6,3\%; 8,3\%; 8,3\%; 12,4\% e 20\%. Além da perda na produção na produção de leite, foram calculados os custos decorrentes do descarte de animais por esterilidade, abortamento, mortalidade perinatal, reposição de animais e aumento no intervalo de partos. A média dos valores anuais de perdas econômicas das propriedades testadas foi de $\mathrm{R} \$ 14.747,92$ (US\$ 3.546,79), com variação entre as propriedades estudadas de $\mathrm{R} \$$ 9.586,25 - R \$ 24.491,87 (US\$ 2.305,44 - US\$5.890,16).

Dos 20 equinos examinados, nenhum foi reagente ao teste do AAT. Das 29 amostras caninas cinco foram reagentes ao teste de triagem (AAT), entretanto nenhuma delas reagiu ao teste confirmatório (2-ME).

A população humana no município de Flor do Sertão é de 1.594 habitantes. Foram examinadas 206 (12,9\%) pessoas para brucelose no município (120 mulheres e 86 homens). Foram positivas para brucelose 11 pessoas, sendo sete mulheres e quatro homens. A prevalência total de brucelose entre os examinados foi de 5,33\% (11/206); para mulheres foi de 5,83\% (7/120) e para homens 4,65\% (4/86). Neste estudo, 72,7\% (8/11) dos pacientes confirmados para a doença estiveram expostos ao fator de risco contato ocupacional, por se tratarem de produtores de leite que mantém contato direto com bovinos. 54,5\% (6/11) pacientes tem idade igual ou superior a 60 anos, e 63,6\% (7/11) são mulheres. Quanto ao local de residência, 72,7\% (8/11) dos pacientes são residentes na zona rural e 27,3\% (3/11) na zona urbana. Os pacientes positivos residentes na zona rural estão localizados em quatro localidades do interior do município.

Para a espécie bovina, foram testadas propriedades em nove localidades distintas, sendo que os positivos se concentraram em três dessas localidades. Os equinos examinados, foram oriundos de nove localidades, embora nenhum foi 
positivo. Os cães testados foram oriundos das três localidades em que os bovinos foram positivos. Os cinco cães reagentes ao teste de triagem foram residentes em duas localidades.

Na Figura 1, as localidades do Município são representadas de A a J, onde se observa a concentração de bovinos e humanos positivos, e de cães reagentes ocorrendo de maneira sobreposta.

Figura 1 - Distribuição dos casos humanos e bovinos confirmados e de cães reagentes para brucelose (Brucella abortus) em Flor do Sertão em 2017 e 2018.

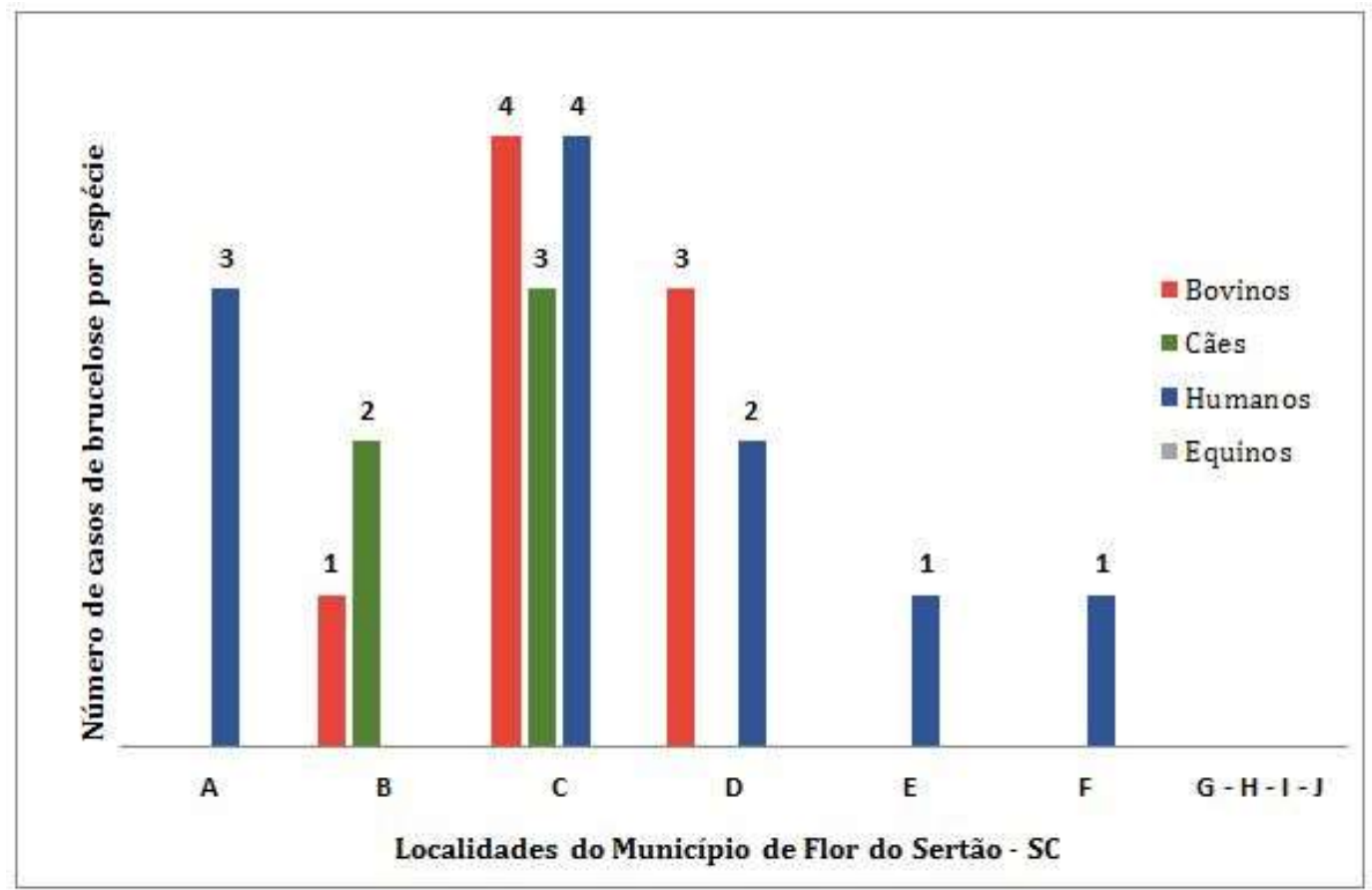

Fonte: Arquivo Pessoal.

Houve correlação positiva $(\mathrm{r}=0,621)$ entre os casos bovinos e humanos relativos às nove localidades $(\mathrm{P}<0,05)$. Foi verificada diferença estatística entre a prevalência de brucelose em bovinos $(0,29 \%)$ e humanos $(5,33 \%)(\mathrm{P}<0,0001)$.

Em relação aos fatores de risco para a doença obtidos a partir de questionário, a análise demonstra que propriedades que fazem o consumo de mais de dois produtos lácteos crus e que apresentam prevalência superior a zero, apresentaram maior probabilidade de serem positivas para a brucelose. Além do risco de serem positivas, representaram maior problema de saúde pública, uma vez que tem como característica o consumo elevado de derivados lácteos. A árvore de decisão apresentou esta dinâmica com $97,7 \%$ de confiança (Figura 2). 
Figura 2 - Diagrama da árvore de decisão da associação de fatores de risco para humanos e da positividade de rebanhos bovinos de Flor do Sertão - SC para brucelose bovina.

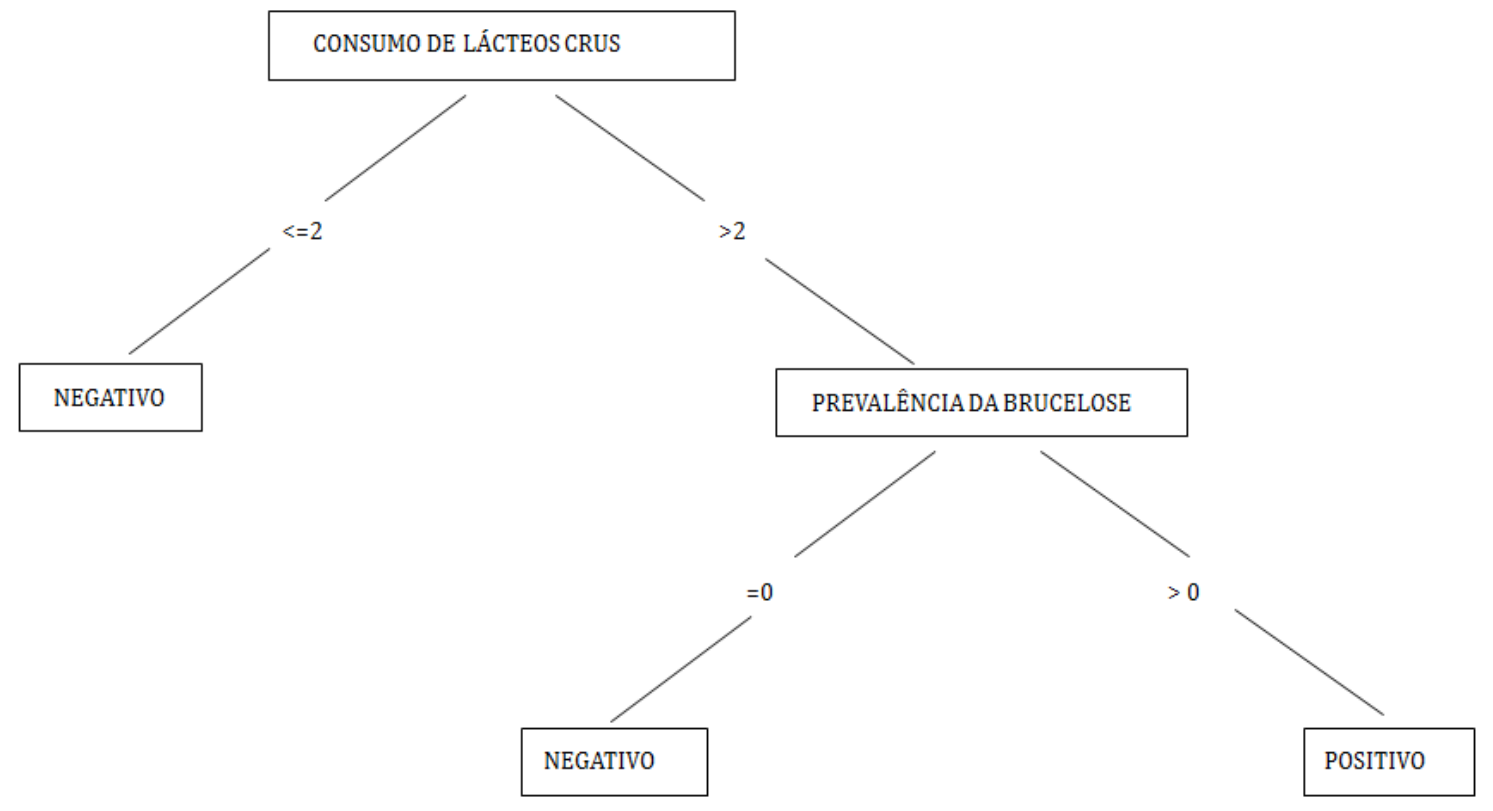

Fonte: Arquivo Pessoal

\section{Discussão}

A prevalência total $(0,29 \%)$ de bovinos com brucelose encontrada neste estudo é considerada baixa. Esse valor é indicativo de que a doença não está amplamente disseminada nos animais, porém, encontra-se disseminada entre os rebanhos do município, conforme a prevalência de focos $(4,58 \%)$. A realização periódica de testes para a doença pode ser considerada determinante para que o número de animais doentes no mesmo foco não seja elevado. A prevalência para animais encontrada neste estudo é superior à encontrada para o Estado de Santa Catarina, por Sikusawa, et al., (2009) de 0,06\%, e inferior à prevalência média para o estado, onde Baumgarten, et al. (2016), relataram prevalência de 1,21\%.

Nos estudos de Sikusawa, et al., (2009) e Baumgarten, et al., (2016), o estado catarinense foi dividido em estratos de acordo com a característica produtiva. Para a Região Oeste, onde se situa o município de Flor do Sertão, a prevalência de brucelose animal estimada foi de 0\% (sem observações) (Sikusawa, et al., 2009) e 1,97\% (Baumgarten, et al., (2016). A relação da prevalência encontrada neste estudo para o estrato da região Oeste em estudos anteriores, se comporta de maneira semelhante à comparação feita para o Estado todo.

Estudos realizados a partir do Programa Nacional de Controle e Erradicação da Brucelose e Tuberculose (PNCEBT), demonstraram prevalência crescente entre os estados, para focos e para animais, apresentando no ano de 2009 os valores: Santa Catarina (0,32\% e 0,06\%) (Sikusawa, et al., 2009); Rio Grande do Sul (2,1\% e 1\%) (Marvulo, et al., 2009); Paraná (4\% e 1,7\%) (Dias, et al, 2009a); Bahia (4,2\% e 0,66\%) (Alves, et al., 2009); Minas Gerais (6\% e 1,1\%) (Gonçalves, et al., 2009); Espírito Santo (9\% e 3,5\%) (Azevedo, et al., 2009); São Paulo (9,7\% e 3,8\%) (Dias, et al., 2009b); Sergipe (12,6\% e 3,4\%) (Silva, et al., 2009); Rio de Janeiro (15,4\% e 4,1\%) (Klein-Gunnewiek, et al., 2009); Goiás (17,5\% e 3\%) (Rocha, et al., 2009); Tocantins (21,2\% e 4,4,\%) (Ogata, et al., 2009); Rondônia (35,2\% e 6,2\%) (Villar, et al. 2009); Mato Grosso (41,2\% e 10,2\%) (Negreiros, et al., 2009); Mato Grosso do Sul (41,5\% e sem informação) (Chate, et al., 2009).

Análises amostrais posteriores, divulgadas por alguns estados em 2016, demonstraram que a brucelose continua endêmica no Brasil, apesar das estratégias do PNCEBT, sendo que o Estado de Santa Catarina continua com baixa prevalência. 
O número de prevalência de focos e animais para o Santa Catarina é de 0,912\% e 1,21\%, respectivamente (Baumgarten, et al., 2016), seguido por: Rio Grande do Sul (3,54\% e 0,98\%) (Silva, et al., 2016), Minas Gerais (3,59\% e 0,81\%) (Oliveira, et al., 2016), Pernambuco (4,5\% e 1,4\%) (Almeida, et al., 2016), Paraíba (4,6\% e 2,5\%) (Clementino, et al., 2016), Espírito Santo (9,3\% e 3,8\%) (Anzai, et al., 2016), São Paulo (10,2\% e 2,4\%) (Dias et al., 2016), Rondônia (12,3\% e 1,9\%) (Inlamea, et al., 2016), Mato Grosso (24\% e 5,1\%) (Barddal, et al., 2016) e Mato Grosso do Sul (30,6\% e 7\%) (Leal Filho, et al., 2016).

Com relação a prevalência de focos/propriedades, o valor encontrado neste estudo (4,58\%), é superior ao estudo de Sikusawa, et al. (2009) para o Estado de Santa Catarina (0,32\%) e para a região Oeste (0,25\%); e de Baumgarten, et al. (2016) para o Estado $(0,91 \%)$ e para a região Oeste $(1,09 \%)$.

A maior prevalência de focos em relação à prevalência em animais encontrada nesta pesquisa discorda do estudo de Baumgarten, et al., (2016). Este fenômeno pode ser justificado devido ao modelo de estudo e ao método de coleta de dados. No estudo de Baumgarten, et al., (2016) foram colhidas amostras aleatórias, com base em cálculo amostral; na pesquisa atual, 35,7\% das propriedades foram testadas. Além do maior número de propriedades testadas, as mesmas são caracterizadas por um pequeno número de animais. Foi observado ainda, que nenhum dos rebanhos positivos apresentou número elevado de animais positivos.

Ainda, todas as propriedades positivas em Flor do Sertão, utilizam o sistema de manejo extensivo. As criações extensivas são mais predispostas a contraírem a doença. Nesse sistema, há maior exposição e compartilhamento de fontes de água e pastagens que podem ser contaminadas com secreções de origem reprodutiva, além da falta de medidas de biosseguridade, que expõe ao contato próximo animais de rebanhos distintos (Miller, et al., 2015; Mota, et al., 2016; Kothalawala, et al., 2017; Cárdenas, et al., 2019). Por outro lado, criações intensivas agrupam mais os animais, o que poderia favorecer a disseminação da doença entre os animais do mesmo rebanho (Santos, et al., 2007). Esta característica justifica a baixa prevalência da doença dentro de um mesmo rebanho, e, consequentemente, a alta prevalência de focos, visto que as localidades que concentram os focos de brucelose no Município são vizinhas.

A maioria dos animais testados foram fêmeas (97,3\%). Fêmeas são a maioria dos animais testados (Miller, et al., 2015) e também mais predispostas a serem soropositivas (Akinseye, et al., 2016). Tal fenômeno se deve a característica produtiva voltada a produção de leite, em que fêmeas são mais numerosas nos rebanhos e permanecem por mais tempo nas propriedades, proporcionando maior exposição ao patógeno, e por sua vez, servem como fonte de infecção para outros animais. Além disso, fêmeas são abatidas apenas quando há redução no desempenho produtivo ou capacidade reprodutiva, quando seu risco de exposição é alto (Akinseye, et al., 2016).

Com relação ao sexo, o estudo proposto diverge em relação à maior prevalência de machos em relação às fêmeas (Akinseye, et al., 2016). A prevalência superior em machos encontrada em Flor do Sertão se deve a pequena proporção de testados, desta maneira, um único animal positivo representa alta taxa de prevalência.

A prevalência de animais positivos por propriedade, apesar de variável $(0,84 \%$ - $8 \%)$ representa perdas econômicas significativas nas propriedades foco, principalmente considerando a característica de propriedades pequenas. $\mathrm{O}$ valor médio de perdas econômicas é ainda superior ao encontrado por Lucas, (2006), que calculou prejuízo anual de $\mathrm{R} \$ 4.279,94$ decorrentes da brucelose, correspondendo a prejuízos anuais de 5\% nas propriedades foco da doença. Em 2013, a brucelose no Brasil gerou um prejuízo estimado em $R$ \$1,005 bilhão, onde cada fêmea adulta positiva representa um prejuízo de $R$ \$473,50 em rebanhos leiteiros e R \$255,20 para rebanhos de corte. Acredita-se ainda, que os valores de perdas são ainda maiores que as estimativas demonstram, uma vez que o valor atribuído aos animais ao nascer no Brasil, são baixos, e, portanto, prejuízos decorrentes de abortamentos e mortalidade perinatal devem ser maiores.

A brucelose vem causando um impacto importante na produção agropecuária desde que a doença foi descoberta. Os prejuízos anuais causados pela doença foram estimados em 32 milhões de dólares, computando apenas as perdas decorrentes 
da redução da produção de leite e de abortos (Brasil, 1971). Mais recentemente as perdas econômicas foram de US\$ 448 milhões no Brasil (Santos, et al., 2013) e US\$ 3,4 bilhões na Índia (Cárdenas, et al., 2019). Outra estimativa recente e aponta que as perdas econômicas causadas pela brucelose bovina no Brasil, supere os valores de 420,12 e 226,47 reais para cada vaca com finalidade leiteira e de carne infectadas, respectivamente (Clementino; Azevedo, 2016), enquanto este estudo demonstra valores de perdas econômicas de 1.843,49 por animal positivo. Na América Latina, estimativas oficiais indicam perdas anuais por brucelose bovina de valores aproximados de US\$ 600 milhões (Seleem, Boyle \& Sriranganathan, 2010).

A ocorrência da brucelose, além de perdas decorrentes da queda da produção animal, incorre em imposição de barreiras sanitárias e restrições comerciais que reduzem a competitividade no mercado de produtos e origem animal (Lucas, 2006; Zhang, et al., 2018). O agronegócio brasileiro, bem como o catarinense, requer a adoção de estratégias focadas em menor custo de produção, maior qualidade de produtos de origem animal e sanidade animal para assegurar comércio perante ao mercado internacional (Lucas, 2006).

As perdas econômicas mundiais devido à brucelose não se limitam a produção animal, são representativas também na saúde pública, envolvendo os custos de tratamento dos pacientes e perda de produtividade das pessoas doentes (Seleem; Boyle; Sriranganathan, 2010). Singh; Dhand e Gill, (2015) relataram que mais estudos devem ser realizados para calcular perdas econômicas, uma vez que as perdas devido à brucelose em animais selvagens não podem ser estimadas, além das grandes perdas de saúde, psicológicas e socioeconômicas que ocorrem e não são consideradas.

Pesquisas em saúde animal que envolvem efeitos financeiros são necessárias para determinar custo/ benefício da implantação de programas de controle e erradicação (Zhang, et al., 2018). Os programas de controle e/ou erradicação normalmente apresentam custos inferiores aos prejuízos econômicos causados pela ocorrência da doença, justificando o investimento em medidas preventivas (Roth, et al., 2003). Estima-se que para cada dólar investido em erradicação são economizados sete dólares em perdas econômicas (Seleem, Boyle \& Sriranganathan, 2010).

Dos equinos testados neste estudo, nenhum foi positivo nos testes. Santos et al., (2016), encontraram uma baixa frequência $(0,26 \%)$ de equinos positivos dentre 767 animais do Paraná, Santa Catarina e Rio Grande do Sul. Em Minas Gerais, foi encontrada prevalência de brucelose em equinos de 1,37\% para animais e 4,28\% para rebanhos (Junqueira, et al., 2015). Na Paraíba, 0,8\% dentre 123 cavalos de trabalho testados foram positivos (Antunes, et al., 2013). É possível inferir, que em regiões em que a brucelose bovina é endêmica, pode haver resposta sorológica em equinos, devido ao compartilhamento de pastagens, fontes e água e contato direto (Junqueira, et al., 2015; Santos et al., 2016).

Existem evidências de que um equino positivo para brucelose, com abscessos recorrentes, seja capaz de transmitir a doença para bovinos e cães (Junqueira, et al., 2015). Apesar da dificuldade de estabelecer a transmissão da doença na espécie, não se pode desprezar o potencial de transmissão da infecção para outros animais e para humanos, o que caracteriza um papel importante também na saúde pública (Corbel, 2006). Além disso, em estados como Santa Catarina, cuja prevalência da doença em bovinos é baixa, esse potencial deve ser cuidadosamente controlado para que não interfira na erradicação da doença (Santos, et al., 2016).

Embora cinco cães reagiram para brucelose no teste de triagem, nenhum foi positivo ao teste confirmatório. Os testes rápidos de aglutinação podem reagir de forma cruzada com anticorpos para Bordetella spp., Pseudomonas spp., Moraxella spp., Yersinia spp., Salmonella spp. e outras bactérias Gram-negativas (Makloski, 2011). Tais bactérias apresentam semelhança antigênica com B. abortus e podem induzir anticorpos que reagem de forma cruzada com antígenos preparados a partir do LPS em testes de diagnóstico (Adone \& Pasquali, 2013). O teste do 2-ME reduz essa reação cruzada através do rompimento de pontes dissulfeto das imunoglobulinas $\mathrm{M}$ e passa a detectar apenas as imunoglobulinas da classe $\mathrm{G}$, que são resistentes a ruptura, aumentando a especificidade de detecção de anticorpos anti-Brucella abortus (Minharro, et al., 2005; Seleem; Boyle; Sriranganathan, 2010). 
Dorneles, et al., (2011) testaram 374 soros de cães para B. abortus no Tocantins, e destes dois reagiram ao teste de triagem, porém o resultado confirmatório também foi negativo. Em Minas Gerais 2,8\% dos cães testados para B. abortus foram positivos, sendo que 18,1\% haviam reagido ao teste de triagem (Almeida, et al., 2004). O fato de os cães não serem positivos a Brucella abortus é um indicativo de que a infecção e disseminação devido ao contato com bovinos não ocorre com facilidade, uma vez que as amostras foram colhidas nas propriedades foco e perifoco. Todavia, não se pode descartar a sua importância no ciclo da doença como transmissor mecânico por transportar placentas, restos de aborto e materiais contaminados (Almeida, et al., 2004; Corbel, 2006). Além disso, Brucella abortus tem potencial de infectarem cães através da ingestão de membranas ou fluidos fetais ou de feto abortado (Makloski, 2011). Quando infectados, os cães eliminam a bactéria através de corrimento vulvar por semanas após o aborto ou parto (Baek, et al., 2003; Makloski, 2011). Além disso, cães cronicamente infectados podem ser negativos na sorologia e ainda assim, manter o agente infeccioso alojado em gânglios linfáticos, fígado, baço e próstata (Baek, et al., 2003; Makloski, 2011).

A prevalência humana elevada $(5,33 \%)$ encontrada destaca o papel da brucelose na saúde pública e os cuidados com fatores de risco. No mundo todo, cerca de 500.000 novos casos humanos de brucelose ocorrem a cada ano (Pappas, et al., 2006). A distribuição geográfica da doença está em constante mudança devido a emergência e novos focos e reemergência de antigos (Seleem, Boyle \& Sriranganathan, 2010). Uma casuística importante de brucelose humana é percebida principalmente na Ásia Central e em países do Oriente Médio (Pappas, et al., 2006). Alguns países têm relacionado às prevalências da brucelose humana a animal, fornecendo subsídios de que animais infectados são fontes de infecção para a brucelose humana (Tumwine, et al., 2015; Lai, et al., 2017).

63,3\% dos humanos positivos foram mulheres, em uma relação aos homens próxima a 2:1. Esta informação discorda de Corbel, (2006), que relata que a maioria dos casos humanos é em homens. Outros estudos também demonstraram maior proporção em homens 74\% (Lai, et al., 2017); 67\% (Chalabiani, et al., 2019); 73,9\% (Miller et al., 2015), 77,3\% (Rahamathula, 2019) disparidade que é justificada pelos homens estarem mais envolvidos no manejo dos animais de fazenda em relação às mulheres, além da maior exposição ao risco de manipulação de produtos cárneos e lácteos (Akhvlediani, et al., 2017; Rahamathula, 2019). A maior ocorrência da doença em mulheres é coerentes com Soares et al., (2015), cujo estudo no estado de Alagoas, encontrou prevalência humana de brucelose de 4,4\%. Destes, $85 \%$ eram do sexo feminino e $15 \%$ do sexo masculino. O estudo demonstrou maior prevalência no sexo feminino pelo fato de os homens procurarem menos o posto de saúde (Soares, et al., 2015). Em Flor do Sertão, as mulheres de fato buscaram mais o atendimento de saúde, representando $58,2 \%$ do total de examinados (120/206). Desta maneira, a prevalência em homens pode ser superior a encontrada, pois há uma menor procura para a realização de atendimento clínico e/ou exames.

A idade média das pessoas com a doença encontrada por Soares, et al., (2015) foi de 20 e 64 anos. Embora este intervalo de idade seja grande, no seu limite superior, a faixa de idade se aproxima com aquela encontrada no Município de Flor do Sertão, onde 54,5\% das pessoas acometidas tinham idade superior 60 anos. Outros trabalhos apontam outra faixa etária para maior prevalência da doença: de 20 e 45 anos (Corbel, 2006); 44 anos (Lai, et al., 2017); de 20 a 39 anos (Rahamathula, 2019); de 20 a 29 anos (Mufinda, Boinas \& Nunes, 2017); de 20 a 39 anos (Chalabani, et al., 2019); de 21 a 40 anos (Akhvlediani, et al., 2017); 45 anos (Angel, et al., 2012). Os autores justificam que esta é a faixa etária onde se encontra a maior parte da população ativa (20 a 45 anos), a qual está exposta aos riscos do contato com animais domésticos e atividades em açougue. Entretanto, a faixa etária idosa, está exposta ao risco do consumo de leite e derivados crus, devido ao fato que idosos tendem a consumir maior volume destes produtos. Além disso, é possível que as pessoas acima de 60 anos tenham recebido diagnóstico neste período, porém estarem cronicamente infectadas por um contato mais antigo com a brucelose.

Dos pacientes positivos, $100 \%$ (11/11) relataram que consumiram produtos considerados de risco, definidos como leite e derivados crus. Isso esclarece que a doença é predominante associada ao consumo de produtos sem tratamento térmico 
(Tumwine, et al., 2015). Acredita-se, que a venda informal de produtos lácteos sem pasteurização diretamente ao consumidor e casos importados de outras localidades possa ter interferido na dinâmica da doença (Mailles, et al., 2016). Este padrão de consumo de produtos de risco dentre os positivos pode ser verificado em estudos anteriores em outros locais (Miller, et al., 2015; Soares, et al., 2015; Akhvlediani, et al., 2017; Rahamathulla, 2019; Dadar, Shahali \& Whatmore, 2019).

Além dos hábitos de consumo alimentar, o estudo desenvolvido em Flor do Sertão - SC demonstrou o contato ocupacional como fator de risco importante dentre os positivos, dos quais 72,7\% relataram contato direto com animais, de acordo com outros estudos (Kutlu, et al., 2014; Miller, et al., 2015; Singh; Dhand; Gill, 2015; Tumwine, et al., 2015; Akhvlediani, et al., 2017; Lai, et al., 2017; Rahamathulla, 2019; Dadar, Shahali \& Whatmore, 2019; Lindahl, et al., 2019). No estudo de Soares et al., (2015), apenas 5\% dos positivos eram trabalhadores rurais, divergindo dos demais, embora a pesquisa teve como público alvo pessoas residentes em zona urbana

Foram identificados como fatores de risco apenas a associação entre o consumo de lácteos sem tratamento térmico e a prevalência da doença $>0$, os quais demonstraram influência em resultar em propriedades positivas. Alguns fatores de risco identificados em outros estudos foram: o hábito de compra de animais (Lindahl, et al., 2019); ausência de política de controle e erradicação da doença (Singh, Dhand \& Gill, 2015); grandes rebanhos, alta frequência de reposição animal, dificuldade de manejo individualizado de animais e problemas de controle sanitário (Mota et al., 2016). Assistência veterinária, uso exclusivo de inseminação artificial (Mota, et al., 2016), vacinação de rotina, limpeza do piso, conhecimento do proprietário (Lindahl, et al., 2019), pasteurização do leite e rigorosas políticas de vigilância e controle em toda a produção de lácteos crus foram caracterizados como fatores protetores para a doença (Mailles, et al., 2016; Dadar, Shahali \& Whatmore, 2019).

Na figura 1, percebe-se que ocorre uma sobreposição espacial dentre os humanos e bovinos positivos e cães reagentes para brucelose. Acredita-se que a ocorrência de brucelose humana deve seguir os casos da brucelose bovina, embora mais estudos seriam necessários para entender as condições que contribuíram para essa ocorrência (Ryu, Magalhães \& Chun, 2019). O risco de humanos positivos para brucelose aumenta com o aumento da prevalência para B. abortus em bovinos (Corbel, 2006; Miller, et al., 2015).

Entretanto, neste estudo, a prevalência humana $(5,33 \%)$ foi superior à prevalência animal $(0,29 \%)$. Na literatura, a relação dos casos entre as espécies ocorre de modo inverso. Para cada caso de brucelose humana são esperados 150 casos em bovinos (Lai, et al., 2017). Acredita-se que a razão para este achado se deve ao comércio informal de lácteos e derivados, a possíveis casos importados da doença, e ainda, que houve casuística elevada da doença em bovinos em anos anteriores com a eliminação desses animais do rebanho, e a demora na obtenção do diagnóstico da doença nas pessoas.

A correlação positiva $(\mathrm{P}<0,05)$ entre os casos humanos e bovinos, verificada neste estudo, indica que o aumento no número de bovinos positivos esteja relacionado ao aumento de humanos positivos para a brucelose. É possível inferir, desta maneira, que a transmissão por contato direto ou indireto, entre pessoas e bovinos, é um fator importante corroborado pela figura de distribuição dos casos nas localidades do município. A diferença entre as prevalências de bovinos e humanos $(\mathrm{P}<0,0001)$ demonstra que embora a prevalência animal $(0,29 \%)$ seja baixa, outras fontes de infecção para os humanos possam estar presentes, sendo responsáveis pela alta prevalência em humanos (5,33\%). Essa diferença pode estar atrelada a casos importados da doença, ou ao consumo de produtos de risco trazidos de outras regiões (Mailles, et al., 2016)

Estudos em outros países também têm verificado correlação positiva entre casos humanos e bovinos (Yoon, et al., 2014; Ryu; Magalhães; Chun, 2019). Outros estudos demonstraram que o risco de positivos em humanos aumentou conforme a prevalência para B. abortus foi crescente em bovinos (Schein, 2006; Miller et al., 2015), indicando que o controle da doença nos bovinos é a melhor forma de reduzir inclusive os casos humanos. 


\section{Conclusão}

Nas condições desse estudo, a prevalência para brucelose bovina detectada pode ser considerada baixa, embora a prevalência de rebanhos tenha sido elevada. Isto indica que a doença está disseminada no Município de estudo, mas com ocorrência intra-rebanhos baixa. Cães e equinos não demonstraram importância para a doença. As perdas econômicas corroboram a importância de desenvolver programas de controle e erradicação para a doença, uma vez que os prejuízos decorrentes dela são representativos, principalmente em pequenas propriedades, como são as de Flor do Sertão. A prevalência da doença em humanos foi elevada, superior à prevalência animal, demonstrando correlação positiva com a ocorrência da doença em bovinos. Dos humanos positivos, a maioria relatou manter contato com animais que são possíveis fontes de infecção. Os fatores de risco associados a positividade da doença, permitem concluir que o consumo de lácteos crus pode ser responsável pela elevada prevalência humana. Estes achados demonstraram que a doença ainda é prevalente em bovinos, assim como em humanos, cujo perfil se mantém sendo ocupacional e associado ao consumo de produtos de risco.

\section{Referências}

Adone, R., \& Pasquali, P. (2013). Epidemiosurveillance of brucellosis. Revue Scientifique et Technique de l'OIE, Paris. 32, (1), 199-205. 10.20506/ rst.32.1.2202.

Aires, D. M. P, Coelho, K. O, Neto Silveira, O. J. (2018). Brucelose bovina: aspectos gerais e contexto nos programas oficiais de controle. Revista Científica de Medicina Veterinária, 10, (30), 1-16. http://faef.revista.inf.br/.

Akhvlediani, T., Bautista, C. T.; Garuchava, N., Sanodze, L., Kokaia, N.; Malania, L., Chitadze, N.; Sidamonidze, K.; Rivard, R.G.; Hepburn, M.J., Nikolich, M.P., Imnadze, P.; Trapaidze, N. (2017). Epidemiological and clinical features of brucellosis in the country of Georgia. PLoS ONE. 12, (1), 1-12. 10.1371/ journal.pone.0170376.

Akinseye, V. O.; Adesokan, H. K.; Ogugua, A. J.; Adedoyin, F. J.; Otu, P. I.; Kwaghe, A. V., Kolawole, N. O.; Okoro, O. J., Agada, C. A., Tade, A.O., Faleke, O.O., Okeke, A. L., Akanbi, I. M., Ibitoye, M. M.; Dipelou, M. O., Dale, E.J., Lorraine, P., Taylor, A. V., Awosanya, E. A., Cadmus, E. O., Stack, J. A., Cadmus, S. I. (2016). Sero-epidemiological survey and risk factors associated with bovine brucellosis among slaughtered cattle in Nigeria. Onderstepoort Journal of Veterinary Research. 83, (1). 10.4102 / ojvr. v83i1.1002.

Alfieri, A. A, \& Alfieri, A. F. (2017). Doenças infecciosas que impactam a reprodução de bovinos. Revista Brasileira de Reprodução Animal. 41, (1), 133139. http://cbra.org.br/br/.

Almeida, A. C., Santorelli, A., Bruzadelli, R. M. Z. \& Oliveira, M. M. N. F. (2004). Soroepidemiologia da brucelose canina causada por Brucella canis e Brucella abortus na cidade de Alfenas, MG. Arquivo Brasileiro de Medicina Veterinária e Zootecnia. Short communication. 56, (2), 275-276. 10.1590/S010209352004000200021.

Almeida, E. C., et al (2016). Prevalence and associated risk factors for bovine brucellosis in the state of Pernambuco, Brazil. Semina: Ciências Agrárias. 37, (5), 3413-3424. 10.5433/1679-0359.2016v37n5Supl2p3413.

Alves, A. J. S., et al (2009). Situação epidemiológica da brucelose bovina no Estado da Bahia. Arquivo Brasileiro de Medicina Veterinária e Zootecnia. 61, 613. 10.1590/S0102-09352009000700002.

Angel, M. O., Ristow, P., Ko, A. I., \& Di-Lorenzo, C. (2012). Serological trail of Brucella infection in an urban slum population in Brazil. The Journal of Infection in Developing Countries. 6, (9), 675-679. 10.3855/jidc.2347.

Antunes, J. M. A. P., et al (2013). Serology for Brucella abortus in cart horses from na urban área in Brazil. Arquivo Brasileiro de Medicina Veterinária e Zootecnia. Short communication. 65, (2), 619-621. 10.1590/S0102-09352013000200044.

Anzai, E. K., Costa, D., Said, A. L. P. R., Grisi-Filho, J. H. H., Amaku, M., Dias, R. A., Ferreira, F., Galvis, J. O. A., Gonçalves, V. S. P., Heinemann, M. B., Telles, E. O., \& Ferreira Neto, J. S. (2016). An update on the epidemiological situation of bovine brucellosis in the state of Espírito Santo, Brazil. Semina: Ciências Agrárias. 37, (5), 3437-3448. 10.5433/1679-0359.2016v37n5Supl2p3437.

Azevedo, S. S., et al (2009). Situação epidemiológica da brucelose bovina no Estado do Espírito Santo. Arquivo Brasileiro de Medicina Veterinária e Zootecnia. 61, 19-26. 10.1590/S0102-09352009000700004.

Baek, B. K., et al (2003). Brucella abortus infection in indigenous Korean dogs. The Canadian Journal of Veterinary Research. Short communication. 67. 312-314. https://www.ncbi.nlm.nih.gov/pmc/articles/PMC280718/.

Barddal, J. E. I., Santos, J. C. Q., Lopes, I. F., Ferreira Neto, J. S., Ferreira, F., Amaku, M., Dias, R. A., Telles, E. O., Grisi-Filho, J. H. H., Heinemann, M. B., Gonçalves, V. S. P., \& Aguiar, D. M. (2016). Effect of vaccination in lowering the prevalence of bovine brucellosis in the state of Mato Grosso, Brazil. Semina: Ciências Agrárias. 37, (5), 3479-3492. 10.5433/1679-0359.2016v37n5Supl2p3479.

Bartoli Meirelles, R. B., Sousa, B. D., \& Mathias, L. A. (2014). Aspectos da brucelose na saúde pública veterinária. PUBVET, 8, (10), 1-30. http://www.pubvet.com.br/. 
Baumgarten, K. D., et al (2016). Prevalence and risk factors for bovine brucellosis in the State of Santa Catarina, Brazil. Semina: Ciências Agrárias, 37, (5), 3425-3436. 10.5433/1679-0359.2016v37n5Sup12p3425.

Brasil, Ministério da Agricultura Pecuária e Abastecimento (1971). Boletim de defesa Sanitária Animal. 5, (1-4). https://www.gov.br/agricultura/ptbr/assuntos/sanidade-animal-e-vegetal/saude-animal/boletins.

Brasil, Ministério da Agricultura, Pecuária e Abastecimento. Manual PNCEBT (Programa Nacional de Controle e Erradicação da Brucelose e Tuberculose Animal). (2006). Brasília, DF. 184.

Cárdenas, L., Awada, L., Tizzani, P., Cáceres, P., \& Casal, J. (2019). Characterization and evolution of countries affected by bovine brucellosis (1996-2014). Transboundary and Emerging Diseases. 1-11. 10.1111/tbed.13144.

Cepea, Centro de Estudos Avançados em Economia Aplicada. Indicador de preços-leite. https://www.cepea.esalq.usp.br/br/indicador/leite.aspx.

Chalabiani, S., Khodadad Nazari, M., Razavi Davoodi, N., Shabani, M., Mardani, M., Sarafnejad, A., \& Akbar Amirzargar, A. (2019). The prevalence of brucellosis in different provinces of Iran during 2013-2015. Iran Journal Public Health. 48, (1), 132-138. https://www.ncbi.nlm.nih.gov/pmc/articles/PMC6401572/.

Chate, S. C., et al (2009). Situação epidemiológica da brucelose bovina no Estado do Mato Grosso do Sul. Arquivo Brasileiro de Medicina Veterinária e Zootecnia. 61, 46-55. 10.1590/S0102-09352009000700007.

Clementino, I. J., et al Azevedo, S. S. (2016). Bovine Bucellosis: epidemiological situation in Brazil and disease control initiatives. Semina: Ciências Agrárias. 37, (4), 2021-2034. 10.5433/1679-0359.2016v37n4p2021.

Clementino, I. J., et al (2016). Epidemiological situation of bovine brucellosis in the state of Paraiba, Brazil. Semina: Ciências Agrárias. 37, (5), 3403-3412. 10.5433/1679-0359.2016v37n5Supl2p3403.

Corbel, M. J. (2006). Food and Agriculture Organization of the United Nations, World Health Organization \& World Organisation for Animal Health. Brucellosis in humans and animals. World Health Organization. http://www.who.int/iris/handle/10665/43597.

Dadar, M., Shahali, Y., \& Whatmore, A. M. (2019). Human brucellosis caused by raw dairy products: a review on the occurrence, major risk factors and prevention. International Journal of Food Microbiology. 292, 39-47. 10.1016/ j. ijfoodmicro.2018.12.009.

Dias, J. A., Müller, E. E., Dias, R. A., Freitas, J. C., Amaku, M., Ferreira, F., Silva, M. C. P., Lôbo, J. R., Figueiredo, V. C. F., Gonçalves, V. S. P., \& Ferreira Neto, J. S. (2019). Situação epidemiológica da brucelose bovina no Estado do Paraná. Arquivo Brasileiro de Medicina Veterinária e Zootecnia, Belo Horizonte, 61, 66-76. 10.1590/S0102-09352009000700009.

Dias, R. A., Belchior, A. P. C., Ferreira, R. S., Gonçalves, R. C., Aguiar, R. S. C. B., Sousa, P. R., Santos, A. M. A., Amaku, M., Ferreira, F., Telles, E. O., Grisi-Filho, J. H. H., Heinemann, M. B., Gonçalves, V. S. P., \& Ferreira Neto, J. S. (2016). Controlling bovine brucellosis in the state of São Paulo, Brazil: results of ten years of vaccination program. Semina: Ciências Agrárias. 37, (5), 3505-3518. 10.5433/ 1679-0359.2016v37n5Supl2p3505.

Dias, R. A., et al (2009). Situação epidemiológica da brucelose bovina no Estado de São Paulo. Arquivo Brasileiro de Medicina Veterinária e Zootecnia. 61, 118-125. 10.1590/S0102-09352009000700015.

Dorneles, E. M.S., et al (2011). Anticorpos anti-Brucella canis e anti-Brucella abortus em cães de Araguaína, Tocantins. Braziian Journal of Veterinary Research and Animal Science. 48, (2) 167-171. 10.11606/S1413-95962011000200010.

Epagri, Empresa de Pesquisa Agropecuária e Extensão Rural de Santa Catarina. CEPA (Centro de Socioeconômica e Planejamento Agrícola). https://cepa.epagri.sc.gov.br/index.php/precos-agricolas-mensais-indice/.

Flor do Sertão. (2017). Lei n 665/2017. Institui o PMCEBT - Programa municipal de controle e erradicação da brucelose e da tuberculose animal, e dá outras providências. Prefeitura Municipal. https://www.diariomunicipal.sc.gov.br/site/?r=site/acervoView\&id=1235552.

Gonçalves, V. S. P., Delphino, M. K. V. C., Dias, R. A., Ferreira, F., Amaku, M., Ferreira Neto, J. S., Porto, T. B., Alves, C. M., Figueiredo, V. C. F., \& Lôbo, J. R. (2009). Situação epidemiológica da brucelose bovina no Estado de Minas Gerais. Arquivo Brasileiro de Medicina Veterinária e Zootecnia. 61, 35-45. 10.1590/S0102-09352009000700006.

IBGE, Instituto Brasileiro de Geografia e Estatística. Censo Agropecuário (2017). https://cidades.ibge.gov.br/brasil/sc/flor-do-sertao/pesquisa/24/76693.

IBGE, Instituto Brasileiro de Geografia e Estatística. Censo Populacional (2010). https://cidades.ibge.gov.br/brasil/sc/.

IBGE, Instituto Brasileiro de Geografia e Estatística. Pesquisa Nacional de Saúde. (2013). https://cidades.ibge.gov.br/brasil/sc/pesquisa/47/0.

IBGE, Instituto Brasileiro de Geografia e Estatística (2016). Produção da Pecuária Municipal. 44, 1-51.

Inlamea, O. F., Rocha, A. B., Ferreira, F., Grisi-Filho, J. H. H., Heinemann, M. B., Dias, R. A., Telles, E. O., Gonçalves, V. S. P., Amaku, M., \& Ferreira Neto, J. S. (2016). Effect of vaccination in lowering bovine brucellosis in the state of Rondônia, Brazil. Semina: Ciências Agrárias. 37, (5), 3493-3506. 10.5433/1679-0359.2016v37n5Sup12p3493.

Junqueira, D. G., et al (2015). Brucellosis in working equines of cattle farms from Minas Gerais State, Brazil. Preventive Veterinary Medicine. 121, (3-4), 380-385. 10.1016/j.prevetmed.2015.06.008.

Klein-Gunnewiek, M. F. C., Amaku, M., Dias, R. A., Ferreira, F., Gitti, C. B., Pereira, L. A., Figueiredo, V. C. F., Lobo, J. R., Gonçalves, V. S. P., \& Ferreira Neto, J. S. (2009). Situação epidemiológica da brucelose bovina no Estado do Rio de Janeiro. Arquivo Brasileiro de Medicina Veterinária e Zootecnia, Belo Horizonte, 61, 77-84. 10.1590/S0102-09352009000700010. 
Kothalawala, K. A. C., et al (2017). Association of farmers' socio-economics with bovine brucellosis epidemiology in the dry zone of Sri Lanka. Preventive Veterinary Medicine. 147, 117-123. 10.1016/ j.prevetmed.2017.08.014.

Kutlu, M., et al (2014). Risk factors for occupational brucellosis among veterinary personnel in Turkey. Preventive Veterinary Medicine. 117, 52-58. 10.1016 /j.prevetmed.2014.07.010.

Lai, S., Zhou, H., Xiong, W., Gilbert, M., Huang, Z., Yu, J., Yin, W., Wang, L., Chen, Q., Li, Y., Um, D., Zeng, L., Ren, X., Geng, M., Zhang, Z., Cui, B., LI, T., Wang, D., Li, Z., Wardrop, N. A., Tatem, A. J., \& Yu, H. (2017). Changing epidemiology of Human Brucellosis, China, 1955-2014. Emerging Infectious Diseases. 23, (2), 184-94. https://wwwnc.cdc.gov/eid/article/23/2/15-1710_article.

Leal Filho, J. M., Bottene, I. F. N., Monteiro, L. A. R. C., Pellegrin, A. O., Gonçalves, V. S. P., Ferreira, F., Dias, R. A., Amaku, M., Telles, E. O., Grisi-Filho, J. H. H., Heinemann, M. B., \& Ferreira Neto, J. S (2016). Control of bovine brucellosis from 1998 to 2009 in the state of Mato Grosso do Sul, Brazil. Semina: Ciências Agrárias. 37, (5), 3467-3478. 10.5433 / 1679-0359.2016v37n5Sup12p3467.

Lindahl, J. F., et al (2019). Risk factors for Brucella seroprevalence in peri-urban dairy farms in five Indians cities. Tropical Medicine and Infectious Disease. 4, (70). $10.3390 /$ tropicalmed4020070.

Lucas, A. (2006). Simulação de impacto econômico da brucelose bovina em rebanhos produtores de leite das regiões Centro-Oeste, Sudeste e Sul. 124p. Tese (Doutorado em Medicina Veterinária) - Universidade de São Paulo, São Paulo. https://www.teses.usp.br/teses/disponiveis/10/10134/tde-26032007-172627/ptbr.php.

Mailles, A., Garin-Bastuji, B., Lavigne, J. P., Jay, M., Sotto, A., Maurin, M., Pelloux, I., O’Callaghan., Mick, V., Vaillant, V., \& De Valk, H. (2016). Human brucellosis in France in the 21st century: Results from national surveillance 2004-2013. Médecine et Maladies Infectieuses. 46, (8), 411-418. 10.1016/j. medmal.2016.08.007.

Makloski, C. L. (2011). Canine Brucellosis Management. Veterinary Clinics of North America: Small Animal Practice. 41, 1209-1219. 10.1016/j. cvsm.2011.08.001.

Marvulo, M. F. V., Ferreira, F., Dias, R. A., Amaku, M., Groff, A. C. M., Gonçalves, V. S. P., Figueiredo, V. C. F., Lobo, J. R., \& Ferreira, Neto J. S. (2009). Situação epidemiológica da brucelose bovina no Estado do Rio Grande do Sul. Arquivo Brasileiro de Medicina Veterinária e Zootecnia. 61, 93-102. 10.1590/S0102-09352009000700012.

Miller, R., Nakavuma, J. L., Ssajjakambwe, P., Vudriko, P., Musisi, N., \& Kaneene, J. B. (2015). The pevalence of brucellosis in cattle, goats and humans in rural Uganda: a comparative study. Transboundary and emerging diseases. 63, (6). 10.1111/tbed.12332.

Minharro, S., et al (2005). Diagnóstico da brucelose canina: dificuldades e estratégias. Revista Brasileira de Reprodução Animal. 29, (3-4), 167-173. http://cbra.org.br/br/.

Mota, A. L. A. A., et al (2016). Large-scale study of herd-level risk factors for bovine brucellosis in Brasil. Acta Tropica. 164. 10.1016/j.actatropica.2016.09.016.

Mufinda, F. C., Boinas, F. B., \& Nunes, C. (2017). Prevalence and factors associated with human brucellosis in livestock professional. Revista de Saúde Pública. 51. (57). 10.1590/s1518-8787.2017051006051.

Negreiros, R. L., Dias, R. A., Ferreira, F., Ferreira Neto, J. S., Gonçalves, V. S. P., Silva, M. C. P., Figueiredo, V. C. F., Lôbo, J. R., Freitas, J., \& Amaku, M. (2009). Situação epidemiológica da brucelose bovina no Estado do Mato Grosso. Arquivo Brasileiro de Medicina Veterinária e Zootecnia. 61, 56-65. 10.1590/S0102-09352009000700008.

Ogata, R. A., Gonçalves, V. S. P., Figueiredo, V. C. F., Lôbo, J. R., Rodrigues, A. L., Amaku, M., Ferreira, F., Ferreira Neto, J. S., \& Dias, R. A. (2009). Situação epidemiológica da brucelose bovina no Estado do Tocantins. Arquivo Brasileiro de Medicina Veterinária e Zootecnia, Belo Horizonte. 61, 126- 134. $10.1590 /$ S0102-09352009000700016.

Oliveira, L. F., Dorneles, E. M. S., Mota, A. L. A. A., Gonçalves, V. S. P., Ferreira Neto, J. S., Ferreira, F., Dias, R. A., Telles, E. O., Grisi-Filho, J. H. H., Heinemann, M. B., Amaku, M., \& Lage, A. P. (2016). Seroprevalence and risk factors for bovine brucellosis in the State of Minas Gerais, Brazil. Semina: Ciências Agrárias.37, (5), 3449-3446. 10.5433/1679-0359.2016v37n5Sup12p3449.

OMS, Organização Mundial de Saúde. (1958). Informe Técnico $\mathrm{n}^{\circ}$ 148. Comitê Mixto FAO/OMS de experts em brucelosis. Genebra, https://apps.who.int/iris/handle/10665/40202.

Pappas, G., Papadimitriou, P., Akritidis, N., Christou, L., \& Tsianos, E. V. (2006). The new global map of human brucellosis. The Lancet Infectious Diseases. 6, 91-99. 10.1016 / S1473-3099 (06) 70382-6.

Pereira, M. G. (2008). Epidemiologia: teoria e prática. Rio de Janeiro: Guanabara Koogan.

Rahamathulla, M. P. (2019). Seroprevalence of human brucellosis in Wadi Al Dawaser region of Saudi Arabia. Pakistan Journal of Medical Science. 35, (1), 129-135. $10.12669 /$ pjms.35.1.55

Rocha, W. V., Gonçalves, V. S. P., Coelho, C. G. N. F. L., Brito, W. M. E. D., Dias, R. A., Delphino, M. K. V. C., Ferreira, F., Amaku, M., Ferreira Neto, J. S., Figueiredo, V. C. F., Lôbo, J. R., \& Brito, L. A. B. (2009). Situação epidemiológica da brucelose bovina no Estado de Goiás. Arquivo Brasileiro de Medicina Veterinária e Zootecnia, Belo Horizonte, 61, 27-34. 10.1590/S0102-09352009000700005.

Roth, F., Zinsstag, J., Orkhon, D., Hutton, G., Cosivi, O., Carrin, G., \& Otte, J. (2003). Human health benefits from livestock vaccination for brucellosis: case study. Bulletin of the World Healh Organization. 81, (12). https://apps.who.int/iris/handle/10665/268863.

Ryu, S., Magalhães, R. J. S., \& Chun, B. C. (2019). The impact of expanded brucellosis surveillance in beef cattle on human brucellosis in Korea: na interrupted time-series analysis. BMC Infectious Disease. 19, (201). https://bmcinfectdis.biomedcentral.com/articles/10.1186/s12879-019-3825-6. 
Research, Society and Development, v. 10, n. 3, e12610313208, 2021

(CC BY 4.0) | ISSN 2525-3409 | DOI: http://dx.doi.org/10.33448/rsd-v10i3.13208

Santos, H. P., et al (2007). Brucelose bovina e humana diagnosticada em matadouro municipal de São Luís - MA, Brasil. Ciência Veterinária nos Trópicos. 10, (2/3), 86-94. http://www.rcvt.org.br/volume10_2_3/86-94.

Santos, R. F., Silva, G. C. P., Assis, N. A., \& Mathias, L. A. (2016). Brucella spp. in equines slaughtered in the South region of Brazil. Arquivo Brasileiro de Medicina Veterinária e Zootecnia. 68, (4), 853-857. 10.1590/1678-4162-8513.

Santos, R. L., Martins, T.M., Borges, A. M., \& Paixão, T.A. (2013). Economic losses due to bovine brucellosis in Brazil. Pesquisa Veterinária Brasileira. 33, (6), 759-764. 10.1590/S0100-736X2013000600012.

Schein, F. B. (2006). Prevalência de Brucelose em rebanhos bovinos leiteiros e ocorrência em trabalhadores rurais do município de Araputanga-MT, Brasil. 2006. 78f. Dissertação (Mestrado em Ciência Veterinária) - Universidade Federal Rural de Pernambuco. Pernambuco.

Seleem, M. N., Boyle, S. M., \& Sriranganathan, N. (2010). Brucellosis: A re-emerging zoonosis. Veterinary microbiology. 140, 392-398. 10.1016 / j.vetmic.2009.06.021.

Sikusawa, S., et al (2009). Situação epidemiológica da brucelose bovina no Estado de Santa Catarina. Arquivo Brasileiro de Medicina Veterinária e Zootecnia. 61,103-108.

Silva, N. S., Groff, A. C. M., Vidor, A. C. M., Grisi-Filho, J. H. H., Heinemann, M. B., Dias, R. A., Telles, E. O., Gonçalves, V. S. P., Amaku, M., Ferreira, F., \& Ferreira Neto, J. S. (2016). Epidemiological situation of brucellosis after implementation of the vaccination program in Rio Grande do Sul State, Brazil. Semina: Ciências Agrárias. 37, (5), 3519-3530. 10.5433/1679-0359.2016v37n5Supl2p3519.

Silva, V. G. S. O., Dias, R. A., Ferreira, F., Amaku, M., Costa, E. L. S., Lôbo, J. R., Figueiredo, V. C. F., Gonçalves, V. S. P., \& Ferreira Neto, J. S. (2009). Situação epidemiológica da brucelose bovina no Estado de Sergipe. Arquivo Brasileiro de Medicina Veterinária e Zootecnia. 61, 109-117. 10.1590/S010209352009000700014

Singh, B. B., Dhand, N. K., \& Gill, J. P. S. (2015). Economic losses occurring due to brucellosis in Indian livestock populations. Preventive Veterinary Medicine. 119, (3-4), 211-215. 10.1016/j.prevetmed.2015.03.013.

Soares, C. P. O. C., et al (2015). Prevalência da Brucella spp em humanos. Revista Latino-Americana de Enfermagem. 23. (5), 919-926. 10.1590/01041169.0350.2632.

Tumwine, G., et al (2015). Human brucellosis: sero-prevalence and associated risk factors in agro-pastoral communities of Kiboga District, Central Uganda. BMC Public Health. 15. https://bmcpublichealth.biomedcentral.com/articles/10.1186/s12889-015-2242-z.

Villar, K. S., et al (2009). Situação epidemiológica da brucelose bovina no Estado de Rondônia. Arquivo Brasileiro de Medicina Veterinária e Zootecnia. 61, 85-92. 10.1590/S0102-09352009000700011.

Yoon, H., et al (2014). Epidemiology of brucellosis among cattle in Korea from 2001 to 2011. Journal of Veterinary Science. 15, (4), 537-543. 10.4142 / jvs.2014.15.4.537.

Zhang, N. Z., Huang, D., Wu, W., Liu, J., Liang, F., Zhou, B., \& Guan, P. (2018). Animal brucellosis control or eradication programs worldwide: a systematic review of experiences and lessons learned. Preventive Veterinary Medicine. 160. 10.1016/ j. prevetmed.2018.10.002. 